\title{
Accurate Evaluation of Charge Asymmetry in Aqueous Solvation
}

\author{
Abhishek Mukhopadhyay \\ Department of Physics, Virginia Tech, Blacksburg, VA 24061, USA \\ Igor S Tolokh \\ Department of Computer Science, Virginia Tech, Blacksburg, VA 24061, USA \\ Alexey V Onufriev ${ }^{\text {}}$ \\ Department of Computer Science, Virginia Tech, Blacksburg, VA 24061, USA and \\ Department of Physics, Virginia Tech, Blacksburg, VA 24061, USA
}

\begin{abstract}
Charge hydration asymmetry (CHA) - a characteristic dependence of hydration free energy on the sign of the solute charge - quantifies the asymmetric response of water to electric field at microscopic level. Accurate estimates of CHA are critical for understanding of hydration effects ubiquitous in chemistry and biology. However, measuring hydration energies of charged species is fraught with significant difficulties, which lead to unacceptably large (up to $300 \%$ ) variation in the available estimates of the CHA effect. We circumvent these difficulties by developing a framework which allows us to extract and accurately estimate the intrinsic propensity of water to exhibit CHA from accurate experimental hydration free energies of neutral polar molecules. Specifically, from a set of 504 small molecules we identify two pairs that are analogous, with respect to $\mathrm{CHA}$, to the $\mathrm{K}^{+} / \mathrm{F}^{-}$ pair - a classical probe for the effect. We use these "CHA-conjugate" molecule pairs to quantify the intrinsic charge-asymmetric response of water to the microscopic charge perturbations: the asymmetry of the response is strong, $\sim 50 \%$ of the average hydration free energy of these molecules. The ability of widely used classical water models to predict hydration energies of small molecules correlates with their ability to predict CHA.
\end{abstract}

\section{INTRODUCTION}

The water molecule, $\mathrm{H}_{2} \mathrm{O}$, is among the simplest chemical structures, yet the liquid phase of water is among the most complex liquids. Many of its unique, vital for Life properties, including the ability to establish complex hydrogen bonded structure [1], are due to the complexity of electrostatic interactions. The ability of water to hydrate ions and biomolecules is crucial to biological functions; detailed atomistic understanding of these functions is impossible without accurate description of the energetics of aqueous solvation (hydration). Given the very polar nature of water as a solvent, understanding key details of molecular hydration requires accurate and comprehensive experimental characterization of electrostatic properties of water molecule in liquid phase at microscopic level. This characterization is currently lacking: for example, even the value of the dipole moment of water molecule in liquid phase is still debated [2]: the experimental range is from 2.7 to $3.2 \mathrm{D}, 3$, 5. and higher multipole moments are not available from experiment at all.

Experiments further lack consensus in quantifying the key characteristic of the asymmetry of water response to microscopic fields of solvated charges - the so called charge hydration asymmetry (CHA) [6 20], Fig. 1] One of its earliest 21] known manifestations is the observed differences in hydration free energies of oppositely

*Electronic address: alexey@cs.vt.edu charged ions of similar size, e.g., the $\mathrm{K}^{+} / \mathrm{F}^{-}$pair, Fig. 1 , completely unaccounted for in the linear response continuum framework 22]. These CHA-related differences are larger than many relevant biomolecular energy scales, such as folding free energy of a typical protein. The CHA effects are strong not only for the charged, but also for net neutral solvated structures [14, 18, 20]. Absolute single-

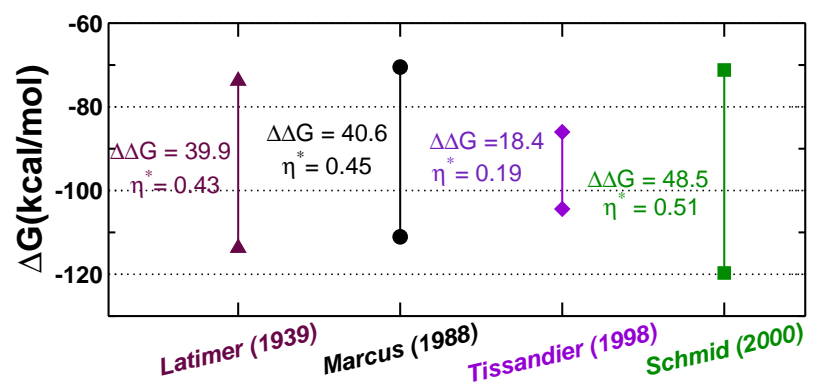

FIG. 1: Uncertainty in experimentally determined charge hydration asymmetry (CHA) for $\mathrm{K}^{+} / \mathrm{F}^{-}$ion pair. Shown are the hydration free energies, $\Delta G$, for $\mathrm{K}^{+}$(top markers) and $\mathrm{F}^{-}$(bottom markers) from four classical experimental references [21, 23 25], from historical to modern, spanning more than eight decades. Also shown are the absolute CHA, $\Delta \Delta G=\Delta G\left(\mathrm{~K}^{+}\right)-\Delta G\left(\mathrm{~F}^{-}\right)$, and the dimensionless relative CHA [16], which quantifies the asymmetry of the charge hydration: $\eta^{*}=\left|\frac{\Delta \Delta G}{\langle\Delta G\rangle}\right|$, where $\langle\Delta G\rangle=$ $\frac{1}{2}\left(\Delta G\left(\mathrm{~K}^{+}\right)+\Delta G\left(\mathrm{~F}^{-}\right)\right)$.

ion solvation free energies are conventionally deduced from experimental estimates of hydration enthalpies of 
ionic solutions via the Born-Haber cycle [24, 25] using highly controversial estimation of absolute proton $\left(\mathrm{H}^{+}\right)$ hydration enthalpy. Uncertainties of up to $16 \mathrm{kcal} / \mathrm{mol}$ are reported that stem from various degrees [26] of extrathermodynamic assumptions [23, 25, 27] or cluster ion solvation approximation [24] made in obtaining these estimates. Furthermore, ion hydration free energy is directly affected by liquid-vapor and liquid-cavity surface (interface) potentials: every time a charge $q$ crosses such interface, the electrostatic energy of the charge changes by $q \phi_{\text {int }}$, where $\phi_{\text {int }}$ is the electrostatic potential difference associated with a the interface. However, available experimental estimates of this elusive quantity can differ by a factor of five 28, 29]; there are fundamental difficulties associated with measuring and even interpreting 30] the water surface potential [31, 32].

The net result of these, and possibly other problems, is an almost $300 \%$ variation between the four available comprehensive experimental data sets of ion hydration energies in the strength of the CHA effect for $\mathrm{K}^{+} / \mathrm{F}^{-}$ pair, Fig. 1 At the same time, even uncertainties of the order of just several $k_{B} T$ can have profound effect on the thermodynamics or kinetics of different phenomena where ion dehydration is involved, e.g., membrane ion channel transport, ion binding to receptor proteins, etc. An accurate quantification of the inherent CHA of water is therefore of paramount importance for accurate quantitative estimates in biology, chemistry, and physics.

In contrast to ions, direct measurements of experimental hydration energies [33, 34] of neutral solutes are relatively straightforward and very accurate, with errors within a fraction of $\mathrm{kcal} / \mathrm{mol}$. The two primary sources of error in the case of ionic solutes are completely eliminated in the case of net neutral solutes, $\sum_{i} q_{i}=0$. First, the contribution of the interface potential to the energy of the solute transfer is zero, $\phi_{i n t} \sum_{i} q_{i}=0$. Second, experimental hydration energy estimates of neutral molecules are typically obtained using calorimetric measurements of transfer free energy, which are free from empirical reference energies such as the proton hydration free energy needed in the case of ions. 35] Connection to computational models is also easy to make since the relationship between measured and computed hydration energies is straightforward for neutral solutes 34, 36 39], in contrast to ions, see e.g. Ref. 38] for a detailed overview of the associated issues. We therefore propose to quantify the CHA effect by utilizing experimental hydration energies of small neutral molecules, rather than ions. Specifically, we identify "CHA-conjugate" pairs of anionlike and cation-like neutral polar molecules that behave just like the $\mathrm{K}^{+} / \mathrm{F}^{-}$pair with respect to charge hydration asymmetry. To characterize the asymmetry of water response to microscopic charge independently of the strength of the charge probe (absolute hydration energies of small neutral molecules are much smaller than those of ions) in what follows we will quantify the strength of the CHA effect by a dimensionless quantity $\eta^{*}=\left|\frac{\Delta \Delta G}{\langle\Delta G\rangle}\right|$, where $\langle\Delta G\rangle$ is the average hydration energy of the pair of solutes, Fig. [1.

\section{RESULTS AND DISCUSSION}

Without loss of generality, the hydration free energy, $\Delta G$, of a molecule can be decomposed into symmetric, $\Delta G^{\text {sym }}$, and asymmetric, $\Delta G^{a s y m}$, parts with respect to inversion of solute charges: $\Delta G=$ $\Delta G^{\text {sym }}+\Delta G^{\text {asym }} ;$ such that upon sign inversion of the partial atomic charges $\Delta G^{\text {sym }}$ remains invariant, whereas $\Delta G^{\text {asym }} \rightarrow-\Delta G^{\text {asym }}$. Physically, the proposed symmetric-asymmetric decomposition of the solvation free energy reflects the inherently charge-asymmetric microscopic response of water to perturbing electric field due to a solvated charge. Here, $\Delta G^{a s y m}$ part accumulates all the deviations from the symmetric response $\Delta G^{\text {sym }}$ that is invariant upon inversion of the perturbing electric field; the response due to dipole polarization of water contributes primarily to the polar part of $\Delta G^{\text {sym }}$. The non-polar component of $\Delta G$ for molecules is also mostly symmetric [14], and is similar in magnitude for molecules of similar size. The magnitude of $\Delta G^{\text {asym }}$ relative to $\Delta G^{\text {sym }}$ characterizes charge asymmetry of the solvent response: e.g. in a hypothetical water consisting of molecules with a perfectly tetrahedral charge distribution this asymmetric response is zero [16].

For an arbitrary pair (A,B) of molecules, the experimental difference in their hydration energies $\Delta \Delta G=$ $\Delta G(A)-\Delta G(B)$ would contain a mix of CHA-related and unrelated components. Here we propose to cleanly isolate the asymmetric part by identifying special pairs of "CHA-conjugate" neutral molecules - molecules with same $\Delta G^{\text {sym }}$, but equal and opposite $\Delta G^{\text {asym }}$; for these special pairs $\Delta G^{\text {sym }}$ would cancel out in the difference, while $\left|\Delta G^{\text {asym }}\right|$ would combine, so that $\Delta \Delta G=$ $\Delta G^{\text {asym }}(A)-\Delta G^{\text {asym }}(B)$ would contain only the CHA effect we seek to quantify.

Since no known experimental method can perform the decomposition of the total solvation energy into chargesymmetric and asymmetric parts, we must resort to theoretical water models to identify such special pairs of molecules. However, once such a pair is identified, the values of $\Delta G^{\text {sym }}$ and $\Delta G^{\text {asym }}$ for each molecule in the A/B pair can be extracted from the experimental hydration energies; $\Delta G^{\text {sym }}=\frac{1}{2}(\Delta G(A)+\Delta G(B))$ and $\left|\Delta G^{\text {asym }}\right|=\frac{1}{2}|\Delta G(A)-\Delta G(B)|$. The implications of the decomposition for development of simplified water models will be discussed below.

Within the widely used family of TIPnP water models [40], the propensity to cause CHA increases [12, 14] progressively from TIP5P to TIP3P 41] to TIP4PEw 42]. In contrast, one of the earliest water models, BNS [43], does not exhibit any CHA: due to its perfectly tetrahedral geometry, charge inversion within this molecule can be emulated by a set of rotations around its oxygen center - a sufficient condition for the absence of 


\section{Perfect Tetrahedron}

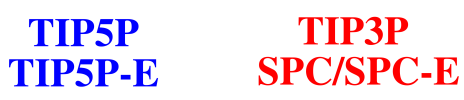

TIP4P-Ew

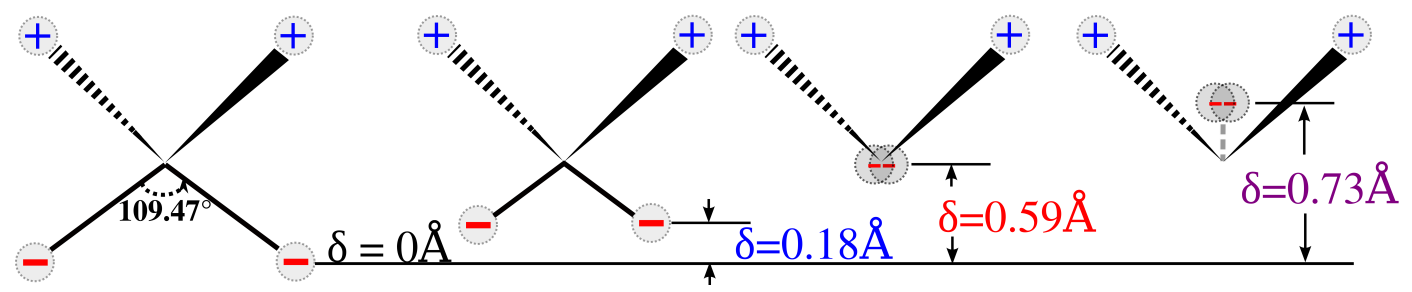

FIG. 2: A family of rigid $n$-point water models arranged in ascending order with respect to deviation from the perfect tetrahedral symmetry (BNS model, zero CHA). The ability of the water models to cause CHA is proportional to the magnitude of parameter $\delta$ that progressively breaks the specific charge inversion symmetry in this family of models.

CHA [16]. This high degree of charge inversion symmetry is progressively broken in other water models shown in Fig. 2 by an order parameter $\delta$ that has an intuitive geometric interpretation. A relation between $\delta$ (geometry) and CHA (energy) is established in SI, where it is shown that $\delta \sim \Omega_{2}$ - the cubic octupole moment of water molecule earlier recognized [16, 44 [46] as key for breaking the charge hydration symmetry. This multipole moment is defined as $\Omega_{2}=(1 / 2)\left(\Omega_{y y z}-\Omega_{x x z}\right)$ where $\Omega_{i j k}$ are the components of the traceless octupole tensor of a water molecule model in the Cartesian frame with origin at the water oxygen center and $\mathrm{OH}$ bonds in the $y z$ plane with $z$-axis bisecting the $\mathrm{H}-\mathrm{O}-\mathrm{H}$ bond angle.

The asymmetric part of the solvation free energy, $\Delta G^{a s y m}$ can be treated as a first order perturbation (with respect to the symmetry breaking variable $\delta$ ) to the completely charge-symmetric part, $\Delta G^{\text {sym }}=\Delta G(\delta=0)$; i.e. $\Delta G(\delta) \simeq \Delta G(\delta=0)+\frac{\partial \Delta G}{\partial \delta} \delta$. For small $\delta$, $\Delta G^{a s y m} \simeq \frac{\partial \Delta G}{\partial \delta} \delta \propto \delta$. To illustrate the idea within a concrete theoretical framework we follow an earlier work [16] where CHA is introduced explicitly into the Born equation [22]:

$$
\Delta G \simeq-\left(1-\frac{1}{\epsilon}\right) \frac{q^{2}}{2\left(R+R_{s}\right)}\left(1-\operatorname{sgn}[q] \frac{\delta}{R+R_{w}}\right) .
$$

Here, $\epsilon$ is the dielectric constant of water, $R_{w}$ is the radius of water molecule, $q$ and $R$ are the ion charge and ionic radius, respectively, and $R_{s}$ is a constant shift to the dielectric boundary [16]. Neglecting a minor difference in the ionic radii of $\mathrm{K}^{+}$and $\mathrm{F}^{-}$ions, 1 results in the same $\Delta G^{\text {sym }}=\Delta G(\delta=0)$ for both ions and a symmetric gap in hydration free energies

$\Delta \Delta G(\delta)=2\left|\Delta G^{a s y m}(\delta)\right|=\left(1-\frac{1}{\epsilon}\right) \frac{q^{2}}{\left(R+R_{s}\right)} \frac{\delta}{\left(R+R_{w}\right)}$,

which increases monotonically with $\delta$, Fig. 3 . In other words, for a cation/anion pair of the same size, 1 describes a "CHA-conjugate" ion pair with identical $\Delta G^{\text {sym }}$ and equal but opposite $\Delta G^{\text {asym }}$.

We propose to use these two distinct characteristics

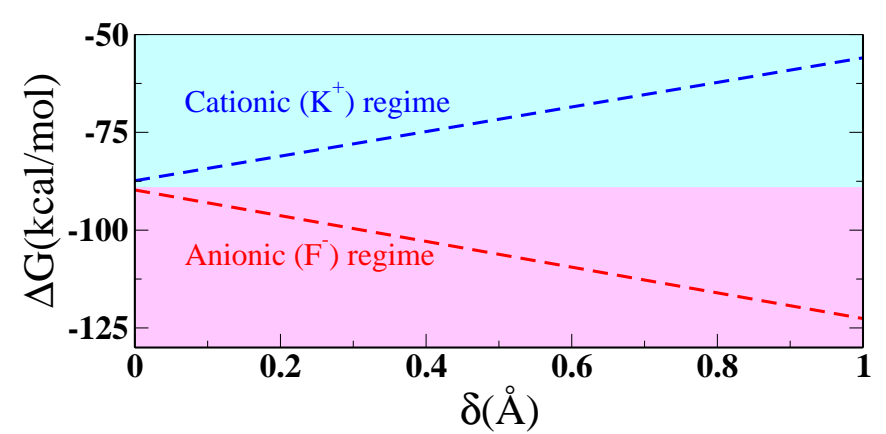

FIG. 3: $\mathrm{K}^{+}$(blue dashed line) and $\mathrm{F}^{-}$(red dashed line) ion pair defines the characteristic behavior of CHA-conjugate pair of solutes - a symmetric, monotonically increasing difference in the electrostatic hydration free energies with respect to increasing degree of the perturbation $\delta$ that breaks the chargeinversion symmetry of the water models. Hydration energies are computed via "CHA-aware" Born equation [16], [1] with $R_{s}=0.52 \AA, R_{w}=1.4 \AA$, and ionic radii from Ref. [47]

of the $\Delta G(\delta)$ for a pair of a "CHA-conjugate" solutes as a signature feature to identify CHA-conjugate pairs among neutral molecules. That is, we seek pairs of small polar neutral molecules for which $\Delta G(\delta)$ behaves similar to that of the $\mathrm{K}^{+} / \mathrm{F}^{-}$pair in Fig. 3. despite differences in scales of hydration free energies - hydration free energies of small neutral molecules are an order of magnitude less than that of the ionic pair. In hydrated polar molecules, for oppositely partially charged atoms one can expect local cation-like or anion-like response of water. The contributions to the overall CHA from the hydration of these atoms, especially atoms of the polar groups of a molecule, may be quite dissimilar. In general, these contributions will not cancel each other completely, resulting in a net CHA effect. In addition to that, in some pairs of molecules, these contributions may combine in a way to produce the $\Delta G(\delta)$ pattern we seek, Fig. 3, Note that the very existence of such pairs is not guaranteed a priori: hydration energy of a molecule, as well as its symmetric and asymmetric parts, are influenced by a complex interplay of screened interactions between its partial charges. 
To illustrate the above points mathematically we use the multi-atomic analog of [1, derived directly from the socalled CHA-GB model [18], 5]in Computational Methods. Within this formalism,

$$
\Delta G(\delta) \simeq-\frac{1}{2}\left(1-\frac{1}{\epsilon}\right) \sum_{i, j} \frac{q_{i} q_{j}}{f_{i j}^{G B}}\left[1-\delta \mathcal{F}_{i j}\right]
$$

where $\frac{1}{f_{i j}^{G B}}=\left(r_{i j}^{2}+R_{i} R_{j} e^{-\frac{r_{i j}^{2}}{4 R_{i} R_{j}}}\right)^{-1 / 2}$ is the purely charge-symmetric Green function of the generalized Born formula [48, 49], and $\mathcal{F}_{i j}=$ $\left[\frac{R_{i} R_{j} e^{-\frac{r_{i j}^{2}}{4 R_{i} R_{j}}}}{f_{i j}^{G B^{2}}}\left(\frac{\operatorname{sgn}\left[\sum_{k} q_{k} e^{-\tau} \frac{r_{i k}^{2}}{R_{i} R_{k}}\right]}{R_{i}-R_{s}+R_{w}}+\frac{\operatorname{sgn}\left[\sum_{l} q_{l} e^{-\tau} \frac{r_{j l}^{2}}{R_{j} R_{l}}\right]}{R_{j}-R_{s}+R_{w}}\right)\right]$. Note that 3 has the same structure as 1 with respect to variation in $\delta$, which further rationalizes the use of our ion-inspired criteria, Fig. 3. for identifying CHA-conjugate pairs among molecules. However, in contrast to the case of a pair of oppositely charged monovalent ions of the same size, for most pairs of neutral molecules their $\left|\Delta G^{\text {asym }}\right|$ are not equal to each other even if their $\Delta G^{s y m}$ are the same. This is due to a complex interplay between the self $\left(\mathcal{F}_{i i}\right)$ and the cross (charge-charge interactions, $\mathcal{F}_{i j}$ ) contributions to CHA within each molecules. In fact, as we show later, we found only two pairs of CHA-conjugate molecules from an available comprehensive diverse set of 504 small, neutral molecules 34] with known experimental hydration free energies (see Computational Methods). Note that these experiments were conducted under conditions to ensure 33] that these molecular solutes were indeed neutral, and therefore avoid the issues related to uncertainties in hydration energies of charged solutes discussed earlier. The fast analytical CHA-GB model [18] was used in the initial screening for the signature $\Delta \Delta G(\delta)$ gap, and the best candidates were then confirmed by careful free energy perturbation (FEP) calculations [34] using the three TIPnP explicit water models in Fig. 2 see Computational Methods.

In the search for CHA-conjugate pairs of small molecules, we utilize rigid, non-polarizable water models of the same geometry (TIPnP family) that have roughly the same dipole moment (less than 0.06 D difference between the models) and exhibit a well studied behavior with respect to $\mathrm{CHA}[14,16]$. It was shown that the different CHA effects these models can produce are proportional to the values of their cubic octupole moments $\Omega_{2}$ and thus to the different values of the symmetry breaking parameter $\delta$ inherent to these models. It is also critical that the monotonic increase of the $\Delta \Delta G(\delta)$ gap with $\delta$ is symmetrical, that is $\Delta G^{s y m}$ is practically the same within this family of water models. These two properties of TIPnP family are essential for the identification of CHA-conjugate pairs of solutes.

Our search has yielded two CHA-conjugate pairs of neutral, but highly polar molecules, Fig. 4, which by our quantitative criteria behave just like the $\mathrm{K}^{+} / \mathrm{F}^{-}$ion pair with respect to CHA, compare Fig. 4 and Fig. 3 . The qualitative rationalization for the unique behavior of these two pairs with respect to CHA is illustrated for one of them in Fig. 5. The anion-like (cationlike) molecule has one relatively highly charged, "CHAdominant" atom, e.g. the amide oxygen of N-methyl acetamide (carboxylic hydrogen of pentanoic acid) that behaves like $\mathrm{F}^{-}$(or $\mathrm{K}^{+}$) with respect to the structuring of water around it. The effect of the opposite charges within each molecule is much more diffuse, and can not cancel the dominant asymmetric contribution from this one atom.

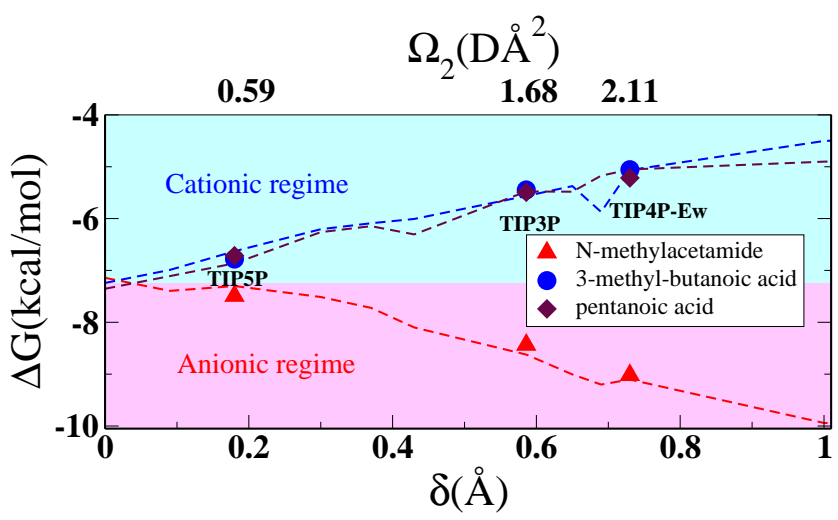

FIG. 4: Hydration free energies of two pairs of molecules analogous to $\mathrm{K}^{+} / \mathrm{F}^{-}$pair with respect to $\mathrm{CHA}$; compare with Fig. 3. The energies are computed using the CHA-GB model (dashed lines) [18], and free energy perturbation (FEP) calculations (solid markers), see Computational Methods. Statistical error bars (not shown) for the FEP calculations are smaller than the symbol size

We quantify the charge hydration asymmetry of these two pairs by their experimental relative CHA, $\eta^{*}=\left|\frac{\Delta \Delta G}{\langle\Delta G\rangle}\right|$. The experimental $\eta^{*}$ for the two CHAconjugate pairs of neutral small molecules (3-methyl butanoic acid, N-methylacetamide) and (pentanoic acid, Nmethylacetamide), respectively, are presented in Table 1 .

TABLE I: Relative CHA, $\eta^{*}$, of two CHA-conjugate pairs of neutral molecules from the experimental hydration free energies 33, 34.

\begin{tabular}{c|c}
\hline Molecule Pair & Experimental relative $\mathrm{CHA}, \eta^{*}$ \\
\hline 3-met Bytanoic acigi & 0.49 \\
\hline
\end{tabular}

The physical meaning of the large, almost 0.5, experimental value of $\eta^{*}$ is that the intrinsic charge- 


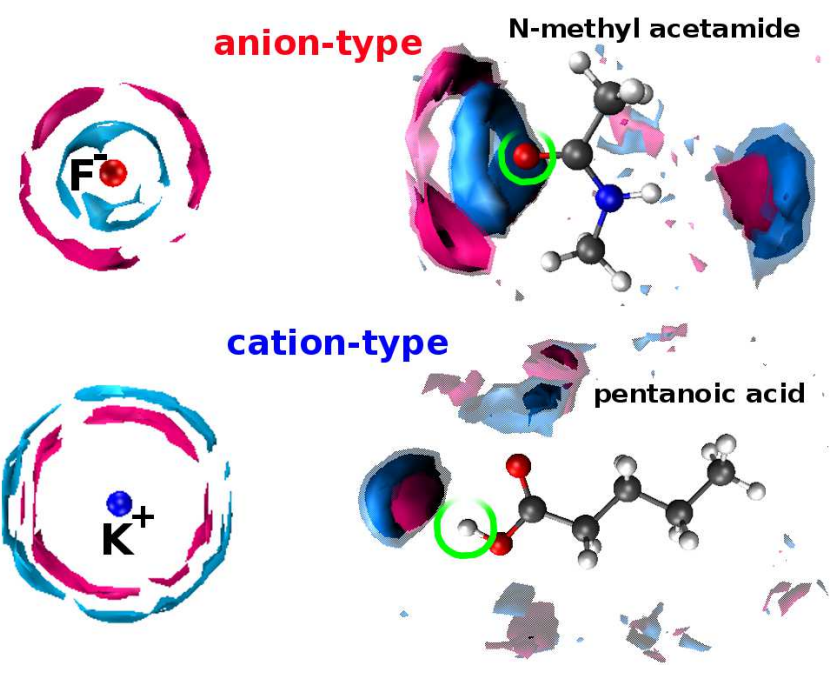

FIG. 5: Similarity between water-structuring effects of ions (left) and one pair of CHA-conjugate neutral polar molecules (right). Shown are water-oxygen (magenta) and waterhydrogen (cyan) occupancy maps computed using TIP3P water model. High probability isomaps are shown using solid surfaces and relatively lower probability isomaps are shown as translucent surfaces. Atom that contributes predominantly to the CHA effect in each molecule is pointed out by green circle. The other cation-like molecule, 3-methyl butanoic acid, is an isomer of pentanoic acid and has similar occupancy isomap, see SI. Using TIP4P-Ew instead of TIP3P results in similar occupancy maps (not shown).

asymmetric response of water to microscopic charges (atomic size CHA perturbations) is strong, comparable to the charge hydration energy itself. For example, experimental hydration energies of pentanoic acid and Nmethylacetamide are -6.16 and $-10.00 \mathrm{kcal} / \mathrm{mol}$ respectively, [33] i.e. the $|\langle\Delta G\rangle|=8.08 \mathrm{kcal} / \mathrm{mol}$ while the $\Delta \Delta G=3.84 \mathrm{kcal} / \mathrm{mol}$ for the pair. In fact, the asymmetry of the individual perturbation caused by a single "CHA-dominant" atom is even stronger, as the net CHA effect in a pair of molecules measured by $\eta^{*}$ can be attenuated somewhat by the opposing contributions from other charges, see Fig. 5 and SI. We again stress that the $\eta^{*}$ from Table $\square$ are free of the issues related to the fundamental or technical difficulties [38] associated with the ion hydration asymmetry: $\eta^{*}$ inferred from CHAconjugate pairs of neutral polar molecules directly and accurately characterize the intrinsic asymmetry of the water response to microscopic electric field. As expected, the uncertainty in the experimental $\eta^{*}$ is small, about 2 $\%$, as seen from the difference between the two CHAconjugate pairs. This high accuracy is in contrast to the almost $300 \%$ difference between available experimental hydration energies for $\mathrm{K}^{+} / \mathrm{F}^{-}$pair, Fig. 1 .

Looking through the prism of CHA at the development of classical fixed-charge water models during the past several decades, we note that, geometrically, the largest variation between the popular water models appears along the single coordinate: one that breaks the perfect charge-inversion symmetry of a perfect tetrahedral charge arrangement assumed by early BNS model, Fig. 2 Judging by the ability of each water model in Table II to predict the experimental $\eta^{*}$, we conclude that to the extent that the charge distribution of a real water molecule in liquid water can be approximated by three point charges, the distribution might be close to the recently proposed water model OPC [50, 51], Fig. 6. This new water model is, perhaps, the very first at-

TABLE II: Relative CHA, $\eta^{*}$, of two CHA-conjugate pairs of neutral molecules from experiment and explicit water simulations. Water models used for comparison are TIP5P, TIP3P, TIP4P-Ew and a recent 4-point model OPC [50, 51]

\begin{tabular}{c|c|c|c|c|c}
\hline Molecule Pair & \multirow{2}{*}{ Exp } & \multicolumn{4}{|c}{ Water models } \\
\cline { 3 - 6 } & & TIP5P & TIP3P & TIP4P-Ew & OPC \\
\hline 3-met Wutanoic acid & & & & & \\
\hline
\end{tabular}

tempt to explicitly consider the CHA effect in water model optimization [51]; the model is curiously successful in accurately reproducing most properties of liquid water [51]. Importantly, the accuracy of the predicted small molecule hydration energies by the water models used in this study (in Table II) correlates well with their ability to reproduce experimental CHA. Namely, RMS errors against experiment in hydration free energy estimates for TIP5P, TIP3P, TIP4P-Ew and OPC are, respectively: $1.85,1.10,1.14$, and $0.97 \mathrm{kcal} / \mathrm{mol}$ (based on 20 neutral molecules, see Computational Methods, chosen [51] to span the entire hydration energy range of the set of 504 neutral molecules). The correlation suggests that the highly accurate experimental $\eta^{*}$ for the CHAconjugate pairs of neutral small molecules can be used to calibrate and improve water models, especially their ability to accurately describe microscopic electrostatic effects important for molecular hydration. The relative quantity $\eta^{*}$ is expected to be insensitive to parameters describing the molecules in "CHA-conjugate" pair (such as the specific parametrization of atomic partial charges) meaning that predicted $\eta^{*}$ mostly characterizes propensity of the water model to cause CHA. This propensity is strong, consistent with the large value of the charge-inversion symmetry breaking separation $\delta$, Fig. 2] comparable to the positive-negative charge separation in water models that approximate the experimental CHA reasonably well, Table II. The CHA strength is also consistent with a relatively large value of the $\Omega_{2}$ octupole moment of water molecule in liquid phase predicted by several independent quantum mechanical estimates [46, 52].

As we have noted before, for each CHA-conjugate molecule pair one can extract $\Delta G^{\text {sym }}$ directly from the experimental hydration free energies, which is essentially the average hydration energy of the pair. This quantity 
can also be used to assess the quality of water models with respect to their ability to describe the symmetric response to solvated charge. For example, for the pairs in Table III. OPC water model gives the best agreement $\Delta G^{\text {sym }} \simeq-7.3 \mathrm{kcal} / \mathrm{mol}$ for each pairs, vs. the experimental value of $\simeq-8 \mathrm{kcal} / \mathrm{mol} ; \Delta G^{\text {sym }}$ for other water models tested here are less accurate, Fig. 4. In the future, it would be interesting to test other water models, including polarizable ones, with respect to their ability to reproduce experimental $\eta^{*}$ and $\Delta G^{s y m}$. We expect that for the current polarizable models, for which the polarizability is added at the dipole level [2, 53 55], one would observe similar $\eta^{*}$ to the corresponding non-polarizable "base" model to which the polarizability is added. This is because the dipole moment response is symmetric with respect to solute charge inversion.

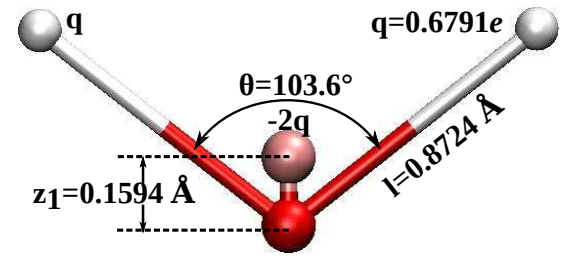

FIG. 6: Charge distribution in OPC water model [50, 51]. The distance between the oxygen center and the positive charges is noticeably shorter than the $\sim 1 \AA$ assumed by common 3 point charge water models 41,42 ].

Having quantified the intrinsic CHA effect of water by $\eta^{*}$ for a pair of neutral, cation-like and anion-like small molecules, we inquire if we can use the accurate experimental value of $\eta^{*}$ to set a lower bound on the value of $\eta^{* i o n}$ for the $\left(\mathrm{K}^{+} / \mathrm{F}^{-}\right)$pair and thus reduce the dramatic uncertainty associated with the original, ionic CHA shown in Fig. 1. To this end, we decompose the molecular $\eta^{*}$ onto individual atomic contributions. To set the bound on $\eta^{*}$ we single out the "CHA-dominant atom" for each molecule, Fig. [5 and use the fact that the CHA effect of these two atoms is larger than that of the combined effect from all the atoms in the molecular pair. This is because of partial cancellation of CHA for neutral molecules. Note that if the CHA-dominant atoms for these CHA-conjugate molecule pairs were exactly of the same size as those of the $\mathrm{K}^{+} / \mathrm{F}^{-}$pair, then $\eta^{*}$ from Table \ could be used directly to set the lower bound on the $\eta^{* i o n}$. In reality, the radii of the CHA-dominant "cation" or "anion" atoms, Fig. 5 , are different from that of $\mathrm{K}^{+}$or $\mathrm{F}^{-}$, see SI. Nonetheless $\Delta \Delta G$ scales with the ion size according to 2, which is consistent with experiment [23 25]. We use this simple scaling relationship to relate atomic (CHA-dominant atoms of the molecules) and ionic $\eta^{*}$, see SI. To obtain more refined estimate of the bound, one needs to consider additional contributions specific to ionic $\Delta \Delta G$, specifically the non-polar interactions estimated in e.g. Ref. [56] and water-vapor interface potential. For the oppositely charged ions, both the liquid-vapor interface potential [30] and the ion cavity potential [57] contribute to $\Delta \Delta G$ : the net contribution is $|2 e| \phi_{\text {int }}$, where $\phi_{\text {int }}$ is the (positive) electrochemical potential corresponding to taking an ion from vacuum to bulk water. Note that the contribution is positive for the positive potential, and thus can only increase $\eta^{* i o n}$ relative to molecular $\eta^{*}$. Experimental estimates of $\phi_{\text {int }}$ range from $+25 \mathrm{mV}$ [28] to $+140 \mathrm{mV}$ [29]. For the lower bound estimate of $\eta^{* i o n}$, taking the lower bound on the experimental estimate of $\phi_{i n t}=25 \mathrm{mV}$, results in a negligible contribution to $\Delta \Delta G$.

The end result is a lower bound of $\eta^{* i o n}\left(\mathrm{~K}^{+} / \mathrm{F}^{-}\right)$set by the molecular $\eta^{*}: \eta^{* i o n}>0.50 \eta^{*}+0.14$. Here, 0.14 is the non-polar contribution to $\eta^{* i o n}$, which, according to a recent theoretical estimate [56] we use here, is not negligible for the $\mathrm{K}^{+} / \mathrm{F}^{-}$ion pair, and originates mostly from the difference in ion-water dispersion interactions for these ions. Substituting $\eta^{*}=0.48$ from Table @ we arrive at:

$$
\eta^{* i o n}\left(\mathrm{~K}^{+} / \mathrm{F}^{-}\right)>0.38
$$

This lower bound estimate suggests that the "original" relative CHA effect for the $\mathrm{K}^{+} / \mathrm{F}^{-}$ion pair, Fig. 1 is also likely to be strong.

To conclude this section, we propose an experiment that can, in principle, estimate $\Delta \Delta G$ and $\eta^{*}$ of $\mathrm{K}^{+} / \mathrm{F}^{-}$ pair accurately, avoiding the uncertainties discussed earlier, Fig. 1. The idea [58] is to measure hydration energies of two net neutral ion pairs, $\mathrm{K}^{+} \mathrm{M}^{-}$and $\mathrm{L}^{+} \mathrm{F}^{-}$, where $\mathrm{L}^{+}$ and $\mathrm{M}^{-}$are relatively large ions (compared to the $\mathrm{K}^{+}$, $\mathrm{F}^{-}$ions) of similar size. For such large ions, $|\Delta G(\mathrm{~L})| \sim$ $|\Delta G(\mathrm{M})| \ll\left|\Delta G\left(\mathrm{~K}^{+}\right)\right|,\left|\Delta G\left(\mathrm{~F}^{-}\right)\right|$. Thus, in very dilute aqueous solutions of $\mathrm{K}^{+} \mathrm{M}^{-}$and $\mathrm{L}^{+} \mathrm{F}^{-}, \Delta G\left(\mathrm{~K}^{+} \mathrm{M}^{-}\right)-$ $\Delta G\left(\mathrm{~L}^{+} \mathrm{F}^{-}\right)=\Delta G\left(\mathrm{~K}^{+}\right)-\Delta G\left(\mathrm{~F}^{-}\right)+\left(\Delta G\left(\mathrm{M}^{-}\right)-\right.$ $\left.\Delta G\left(\mathrm{~L}^{+}\right)\right) \simeq \Delta G\left(\mathrm{~K}^{+}\right)-\Delta G\left(\mathrm{~F}^{-}\right)=\Delta \Delta G\left(\mathrm{~K}^{+} / \mathrm{F}^{-}\right)$. Once $\Delta \Delta G\left(\mathrm{~K}^{+} / \mathrm{F}^{-}\right)$is quantified in this manner, accurate $\eta^{*}=\Delta \Delta G\left(\mathrm{~K}^{+} / \mathrm{F}^{-}\right) /\langle\Delta G\rangle$ can be obtained since the average $\langle\Delta G\rangle$ for the ion pair can be quantified very accurately.

Note that a variation on the above idea might be used to develop an alternative procedure for parameterization of ion force-fields for classical simulations 59 61]. The first step would involve experimental determination of hydration energy of a salt $n \mathrm{~F}^{-} \mathrm{L}^{n+}$ where $\mathrm{L}^{n+}$ is a large "composite" ion such that for its surface atoms the forcefield parameters are well established and are not related to small ion parameters, e.g. $\mathrm{Co}\left(\mathrm{NH}_{3}\right)_{6}^{+3}$ (cobalt hexammine) or $\mathrm{Ni}\left(\mathrm{NH}_{2}\right)_{6}^{+2}$ ion. The next step would be to perform a series of explicit solvent simulations of $n \mathrm{~F}^{-}$ $\mathrm{L}^{n+}$ neutral pair to fit $\mathrm{F}^{-}$parameters to match experimental hydration energy of $n \mathrm{~F}^{-} \mathrm{L}^{n+}$ salt for each water model of interest. Once $\mathrm{F}^{-}$parameters are known, one can perform a set of simulations to obtain cation (e.g. $\mathrm{K}^{+}$) parameters by fitting against known ion pair hydration energies. With the known cation parameters, the procedure can be continued to estimate the parameters for other anions. The oulined protocol would avoid a number of difficulties associated with both experimental and computational[38] estimates of hydration energies of charged species. 


\section{CONCLUSIONS}

In this article we have proposed an approach to use highly accurate experimental hydration energies of small neutral molecules to quantify the effect of charge hydration asymmetry (CHA) - the charge-asymmetric response of water to microscopic electric field. Characteristic dependence of hydration free energies on the sign of charged solutes of similar radii such as the $\mathrm{K}^{+} / \mathrm{F}^{-}$ pair is the best known manifestation of CHA. However, for ion pairs, the quantification of the hydration free energy, and hence the associated CHA, is highly uncertain $(\sim 300 \%$ difference between four available comprehensive sets of experimental data) due to a variety of fundamental, and technical difficulties [24, 26, 30, 38, 62]. Here we overcome these difficulties and accurately quantify CHA by proposing an approach that allows us to separate charge-asymmetric and charge-symmetric parts of the hydration free energies of neutral solutes. The crux of the approach is the identification of pairs of neutral solutes that show charge-asymmetric response of water similar to that that of the $\mathrm{K}^{+} / \mathrm{F}^{-}$pair, based on a set of quantitative criteria we infer from the behavior of $\mathrm{K}^{+} / \mathrm{F}^{-}$pair with respect to the charge-symmetry breaking perturbation. For this purpose we use a combination of "CHA-aware" implicit solvation model and free energy perturbation simulations in different explicit water models to search through a large, comprehensive set of small, neutral drug-like molecules. The search has yielded two "CHA-conjugate" pairs of neutral molecules that behave just like the $\mathrm{K}^{+} / \mathrm{F}^{-}$pair with respect to asymmetric charge hydration. Unlike the corresponding quantity for $\mathrm{K}^{+} / \mathrm{F}^{-}$pair, the measured relative hydration asymmetry of neutral solutes is very accurate. The difference between experimental hydration energies within each pair of these special neutral molecules, relative to the pair's average hydration energy, quantifies the intrinsic charge-asymmetric response of real water, free of extrinsic, complicating factors such as the energetic cost of charge species to cross the liquid/vapor boundary.

Quantitatively, we find the asymmetry of the water response to hydrated microscopic charge (size about $1 \AA$ ) to be close to one-half of the average charge hydration energy of the charge itself, which means that the chargeasymmetry of the intrinsic aqueous response to microscopic fields is strong. Given the paucity of available experimental characteristics of electrostatic properties of water molecule in liquid phase at microscopic level, this result is important.

The availability of a novel, accurate reference value for the charge hydration asymmetry made possible through this work, should be of interest in its own right. For example, we have found that the ability of an explicit water model to predict the correct experimental relative CHA for just one pair of neutral solutes correlates well with the accuracy of the model in predicting absolute hydration energies of small neutral molecules covering a wide range of hydration energies. The observation sug- gests an immediate use of the proposed neutral moleculebased CHA reference for testing, and ultimately improving, solvent models. Note that computational estimate of hydration energies of small neutral solutes is now inexpensive, straightforward and free from serious complicating issues associated with analogous computations for charged species. The inclusion of the proposed accurate CHA reference as an additional optimization target in the process of water model construction may eventually result in more accurate models of water.

\section{COMPUTATIONAL METHODS}

\section{A. The CHA-GB equation}

The implicit solvent hydration free energies were computed using the CHA aware generalized Born equation [18]

$$
\Delta G=-\frac{1}{2}\left(1-\frac{1}{\epsilon}\right) \sum_{i, j} \frac{q_{i} q_{j}}{f_{i j}^{C H A-G B}}
$$

where $q_{i}$ is the charge of atom $i, f_{i j}^{C H A-G B}=$ $\sqrt{r_{i j}^{2}+\tilde{R}_{i} \tilde{R}_{j} e^{-\frac{r_{i j}^{2}}{4 R_{i} R_{j}}}}: R_{i}$ is its effective Born radius, $r_{i j}$ is the spatial separation between atom $i$ and $j$, $\epsilon=80$ is the dielectric constant of water. Here $\tilde{R}_{i}=$ $R_{i}\left(1+\operatorname{sgn}\left[\sum_{j} q_{j} e^{-\tau \frac{r_{i j}^{2}}{R_{i} R_{j}}}\right] \frac{\delta}{R_{i}-R_{s}+\rho_{w}}\right)$ introduces the CHA scaling of the effective Born radii, $R_{i}$ analogous to the CHA scaling of the Born radii in $1 \tau$ is a positive constant $\mathcal{O}(1)$ that controls the effective range of the neighboring charges $(j)$ affecting the CHA of atom $(i)$. The effective Born radii $R_{i}$ are computed using the numerical implementation of the "R6" formula [63], over the dielectric surface (boundary). The later is obtained by increasing 64] the intrinsic atomic radii by $R_{s}=0.52 \AA$, see Ref [18], and further rolling a probe of radius $R_{w}-R_{s}$, where $R_{w}=1.4 \AA$ defines the size of water molecule.

\section{B. Selection of small molecules and identification CHA-conjugate pairs}

To identify CHA-conjugate molecule pairs from the original 504 molecule set [34] we first selected 250 most rigid molecules that undergo very little $(<0.3 \AA)$ conformational change, as seen from molecular dynamics trajectories [65]. We then picked 60 "polar dominant" molecules - those with the smallest nonpolar $\Delta G$ to polar $\Delta G$ ratio $(<0.2)$, based on previous solvation energy estimates [34] in TIP3P water. We first performed single point $\Delta G$ estimates using a CHA-aware implicit solvation model; the polar part was modeled using the CHA-GB equation 5, and the nonpolar part modeled as $\gamma S A S A$, 
where $S A S A$ is the solvent accessible surface area computed numerically [66] and $\gamma=0.005 \mathrm{kcal} / \mathrm{mol} / \AA^{2}$. The topology and coordinates for the molecules were obtained from Ref. 34]. Intrinsic atomic radii set and $\tau$, 5, were optimized (using Nelder-Mead simplex algorithm [67]) against the experimental solvation free energy for the 60 polar molecules using different values of $\delta$ in the range $(0$, $1.0 \AA$ ). For each value of $\delta$ we performed 2000 separate optimizations using random initial guess; the parameter set corresponding to the smallest deviation from the experiments, was used for analysis. The resulting $\Delta G$ for different values of $\delta$ were used to shortlist an initial selection of 11 molecule pairs (17 distinct molecules) that showed promising CHA-conjugate behavior with respect to the $\Delta \Delta G(\delta)$ gap. The initial set was then refined via careful explicit water free energy perturbation calculations in TIP3P, TIP4P-Ew and TIP5P, resulting in two CHA-conjugate pairs, Table \ that exhibit near perfect symmetric, monotonically increasing $\Delta \Delta G(\delta)$ gap.

\section{Explicit solvation free energies}

Molecule topology and coordinate files were prepared in an earlier work [34], using GAFF [68] small molecule parameters assigned by Antechamber 14 [69], and the partial charges were assigned using the Merck-Frosst implementation of AM1-BCC 70]. The hydration free energy calculations in explicit water were performed in GROMACS 4.6.5 71] using standard free energy perturbation (FEP) calculations [34] - the coulomb and van der Waals coupling was reduced from 1 to 0 using 20 intermediate $\lambda$ values. Molecules were solvated in triclinic box with at least $12 \AA$ from the solute to the nearest box edge. Real space electrostatic cutoff was $10 \AA$, and long-range electrostatic interactions were calculated using periodic boundary conditions via. the particle mesh Ewald (PME) summation 72, 73] and all bonds were restrained using the LINCS algorithm. Production simulations were $5 \mathrm{~ns}$ in length at each $\lambda$ value, and free energies and the associated uncertainties were computed using the Bennett acceptance ratio (BAR), namely the gbar feature in GROMACS 4.6.5. The FEP estimates were validated by ensuring convergence of forward (turning the coupling on) and backward (turning the coupling off) computations for two molecules, and also by comparing with the FEP estimates for TIP3P in Ref. [34]. The average errors computed using gbar were roughly 0.05 $\mathrm{kcal} / \mathrm{mol}$ (see SI); the forward and backward computations were comparable with a difference of $0.04 \mathrm{kcal} / \mathrm{mol}$ for the two molecules. The same topology and coordinate files were used along with identical simulation protocol to perform the FEP calculations in each of the four water models, TIP5P, TIP3P, TIP4P-Ew and OPC [50] for 17 molecules (including the 11 shortlisted pairs, above). FEP calculations were also performed for 3 additional molecules, such that the set of these 20 molecules span the range of $\Delta G$ from -0.7 to $-10.0 \mathrm{kcal} / \mathrm{mol}$, which is close to the entire experimental range seen in the set of 504 small neutral molecules. The estimated $\Delta G$ for the set of 20 molecules was used to compare the four water models against the experimental $\eta *$. Their estimated solvation free energies are provided in SI.

\section{Acknowledgments}

This work has been supported by NIH grant R01 GM076121 and, in part, by NSF grant CNS-0960081 and the HokieSpeed supercomputer at Virginia Tech.
[1] Maréchal, Y. The hydrogen bond and the water molecule: The physics and chemistry of water, aqueous and biomedia; Elsevier, 2006.

[2] Leontyev, I. V.; Stuchebrukhov, A. A. Polarizable meanfield model of water for biological simulations with AMBER and CHARMM force fields. J. Chem. Theory Comput. 2012, 8, 3207-3216.

[3] Silvestrelli, P. L.; Parrinello, M. Structural, electronic, and bonding properties of liquid water from first principles. J. Chem. Phys. 1999, 111, 3572-3580.

[4] Sharma, M.; Resta, R.; Car, R. Dipolar correlations and the dielectric permittivity of water. Phys. Rev. Lett. 2007, 98, 247401.

[5] Badyal, Y.; Saboungi, M.-L.; Price, D.; Shastri, S.; Haeffner, D.; Soper, A. Electron distribution in water. $J$. Chem. Phys. 2000, 112, 9206-9208.

[6] Buckingham, A. D. A theory of ion-solvent interaction. Disc. Faraday Soc. 1957, 24, 151-157.

[7] Rashin, A. A.; Honig, B. Reevaluation of the Born model of ion hydration. J. Phys. Chem. 1985, 89, 5588-5593.

[8] Hirata, F.; Redfern, P.; Levy, R. M. Viewing the Born model for ion hydration through a microscope. Int. J. Quantum Chem. 1988, 34, 179-190.

[9] Roux, B.; Yu, H. A.; Karplus, M. Molecular basis for the Born model of ion solvation. J. Phys. Chem. 1990, 94, 4683-4688.

[10] Hummer, G.; Pratt, L. R.; Garcia, A. E. Free energy of ionic hydration. J. Phys. Chem. 1996, 100, 1206-1215.

[11] Bell, R. M. L.; Rasaiah, J. C. From hydrophobic to hydrophilic behaviour: A simulation study of solvation entropy and free energy of simple solutes. J. Chem. Phys. 1997, 10\%, 1981-1991.

[12] Rajamani, S.; Ghosh, T.; Garde, S. Size dependent ion hydration, its asymmetry, and convergence to macroscopic behavior. J. Chem. Phys. 2004, 120, 4457-4466.

[13] Grossfield, A. Dependence of ion hydration on the sign of the ion's charge. J. Chem. Phys. 2005, 122, 024506.

[14] Mobley, D. L.; Barber, A. E.; Fennell, C. J.; Dill, K. A. Charge asymmetries in hydration of polar solutes. $J$. Phys. Chem. B 2008, 112, 2405-14.

[15] Purisima, E. O.; Sulea, T. Restoring charge asymmetry in continuum electrostatics calculations of hydration free 
energies. J. Phys. Chem. B 2009, 113, 8206-8209.

[16] Mukhopadhyay, A.; Fenley, A. T.; Tolokh, I. S.; Onufriev, A. V. Charge hydration asymmetry: The basic principle and how to use it to test and improve water models. J. Phys. Chem. B 2012, 116, 9776-9783.

[17] Bardhan, J. P.; Jungwirth, P.; Makowski, L. Affineresponse model of molecular solvation of ions: Accurate predictions of asymmetric charging free energies. $J$. Chem. Phys. 2012, 137, 124101.

[18] Mukhopadhyay, A.; Aguilar, B. H.; Tolokh, I. S.; Onufriev, A. V. Introducing charge hydration asymmetry into the generalized Born model. J. Chem. Theory Comput. 2014, 10, 1788-1794.

[19] Scheu, R.; Rankin, B. M.; Chen, Y.; Jena, K. C.; BenAmotz, D.; Roke, S. Charge asymmetry at aqueous hydrophobic interfaces and hydration shells. Angew. Chem. Int. Ed. 2014, 53, 9560-9563.

[20] Bardhan, J. P.; Knepley, M. G. Communication: Modeling charge-sign asymmetric solvation free energies with nonlinear boundary conditions. J. Chem. Phys. 2014, $141,131103$.

[21] Latimer, W. M.; Pitzer, K. S.; Slansky, C. M. The free energy of hydration of gaseous ions, and the absolute potential of the normal calomel electrode. J. Chem. Phys. 1939, 7, 108-111.

[22] Born, M. Volumes and heats of hydration of ions. Z. Phys. 1920, 1, 45-48.

[23] Marcus, Y. Ionic radii in aqueous solutions. Chem. Rev. 1988, 88, 1475-1498.

[24] Tissandier, M. D.; Cowen, K. A.; Feng, W. Y.; Gundlach, E.; Cohen, M. H.; Earhart, A. D.; Coe, J. V.; Tuttle, T. R. The proton's absolute aqueous enthalpy and Gibbs free energy of solvation from Cluster-Ion solvation data. J. Phys. Chem. A 1998, 102, 7787-7794.

[25] Schmid, R.; Miah, A. M.; Sapunov, V. N. A new table of the thermodynamic quantities of ionic hydration: values and some applications (enthalpy-entropy compensation and Born radii). Phys. Chem. Chem. Phys. 2000, 2, 97102.

[26] Donald, W. A.; Williams, E. R. Gas-phase electrochemistry: Measuring absolute potentials and investigating ion and electron hydration. Pure Appl. Chem. 2011, 83, 2129-2151.

[27] Hünenberger, P.; Reif, M. Single-ion solvation: experimental and theoretical approaches to elusive thermodynamic quantities; Royal Society of Chemistry, 2011.

[28] Farrell, J. R.; McTigue, P. Precise compensating potential difference measurements with a voltaic cell: The surface potential of water. J. Electroanal. Chem. Interfacial Electrochem. 1982, 139, 37-56.

[29] Fawcett, W. R. The ionic work function and its role in estimating absolute electrode potentials. Langmuir 2008, 24, 9868-9875.

[30] Kathmann, S. M.; Kuo, I.-F. W.; Mundy, C. J.; Schenter, G. K. Understanding the surface potential of water. J. Phys. Chem. B 2011, 115, 4369-4377.

[31] Pethica, B. A. Are electrostatic potentials between regions of different chemical composition measurable? The Gibbs-Guggenheim principle reconsidered, extended and its consequences revisited. Phys. Chem. Chem. Phys. 2007, 9, 6253-6262.

[32] You, X.; Chaudhari, M.; Pratt, L. Comparison of mechanical and thermodynamical evaluations of electrostatic potential differences between electrolyte solutions; Connor
Court Publishing Pty Ltd, 2014; Vol. 400.

[33] Rizzo, R. C.; Aynechi, T.; Case, D. A.; Kuntz, I. D. Estimation of absolute free energies of hydration using continuum methods: accuracy of partial charge models and optimization of nonpolar contributions. J. Chem. Theory Comput. 2006, 2, 128-139.

[34] Mobley, D. L.; Bayly, C. I.; Cooper, M. D.; Shirts, M. R.; Dill, K. A. Small molecule hydration free energies in explicit solvent: An extensive test of Fixed-Charge atomistic simulations. J. Chem. Theory Comput. 2009, 5, 350-358.

[35] Abraham, M. H.; Whiting, G. S.; Fuchs, R.; Chambers, E. J. Thermodynamics of solute transfer from water to hexadecane. J Chem. Soc., Perkin Trans. 2 1990, 291-300.

[36] Roux, B.; Simonson, T. Implicit solvent models. Biophys. Chem. 1999, 78, 1-20.

[37] Mobley, D. L.; Klimovich, P. V. Perspective: Alchemical free energy calculations for drug discovery. J. Chem. Phys. 2012, 137, 230901+.

[38] Lin, Y.-L.; Aleksandrov, A.; Simonson, T.; Roux, B. An overview of electrostatic free energy computations for solutions and proteins. J. Chem. Theory Comput. 2014, 10, 2690-2709.

[39] Li, L.; Fennell, C. J.; Dill, K. A. Field-SEA: A model for computing the solvation free energies of nonpolar, polar, and charged solutes in water. J. Phys. Chem. B 2013, 118, 6431-6437.

[40] Mahoney, M. W.; Jorgensen, W. L. A five-site model for liquid water and the reproduction of the density anomaly by rigid, nonpolarizable potential functions. J. Chem. Phys. 2000, 112, 8910-8922.

[41] Jorgensen, W. L.; Chandrasekhar, J.; Madura, J. D.; Impey, R. W.; Klein, M. L. Comparison of simple potential functions for simulating liquid water. J. Chem. Phys. 1983, 79, 926-935.

[42] Horn, H. W.; Swope, W. C.; Pitera, J. W.; Madura, J. D.; Dick, T. J.; Hura, G. L.; Head-Gordon, T. Development of an improved four-site water model for biomolecular simulations: TIP4P-Ew. J. Chem. Phys. 2004, 120, 9665-9678.

[43] Rahman, A.; Stillinger, F. H. Molecular dynamics study of liquid water. J. Chem. Phys. 1971, 55, 3336-3359.

[44] Kusalik, P. G.; Patey, G. N. On the molecular theory of aqueous electrolyte solutions. II. Structural and thermodynamic properties of different models at infinite dilution. J. Chem. Phys. 1988, 89, 5843-5851.

[45] Te, J. A.; Ichiye, T. Understanding structural effects of multipole moments on aqueous solvation of ions using the soft-sticky dipole-quadrupole-octupole water model. Chem. Phys. Lett. 2010, 499, 219-225.

[46] Niu, S.; Tan, M. L.; Ichiye, T. The large quadrupole of water molecules. J. Chem. Phys. 2011, 134, 134501.

[47] Marcus, Y. Thermodynamics of solvation of ions. J Chem Soc, Faraday Trans 1991, 87, 2995-2999.

[48] Still, W. C.; Tempczyk, A.; Hawley, R. C.; Hendrickson, T. Semianalytical treatment of solvation for molecular mechanics and dynamics. J. Am. Chem. Soc. 1990, 112, 6127-6129.

[49] Onufriev, A. In Modeling Solvent Environments, 1st ed.; Feig, M., Ed.; Wiley: USA, 2010; pp 127-165.

[50] Anandakrishnan, R.; Baker, C.; Izadi, S.; Onufriev, A. V. Point charges optimally placed to represent the multipole expansion of charge distributions. PloS one 2013, 
8, e67715.

[51] Izadi, S.; Anandakrishnan, R.; Onufriev, A. V. Building water models: A different approach. J. Phys. Chem. Lett. 2014, 5, 3863-3871.

[52] Coutinho, K.; Guedes, R.; Cabral, B. C.; Canuto, S. Electronic polarization of liquid water: converged Monte Carlo-quantum mechanics results for the multipole moments. Chem. Phys. Lett. 2003, 369, $345-353$.

[53] Lamoureux, G.; MacKerell, A. D.; Roux, B. A simple polarizable model of water based on classical Drude oscillators. J. Chem. Phys. 2003, 119, 5185-5197.

[54] Yu, H.; van Gunsteren, W. F. Accounting for polarization in molecular simulation. Comput. Phys. Commun. 2005, 172, 69-85.

[55] Wang, J.; Cieplak, P.; Cai, Q.; Hsieh, M.-J. J.; Wang, J.; Duan, Y.; Luo, R. Development of polarizable models for molecular mechanical calculations. 3. Polarizable water models conforming to Thole polarization screening schemes. J. Phys. Chem. B 2012, 116.

[56] Duignan, T. T.; Parsons, D. F.; Ninham, B. W. A continuum model of solvation energies including electrostatic, dispersion, and cavity contributions. J. Phys. Chem. B 2013, 117, 9421-9429.

[57] Ashbaugh, H. S. Convergence of molecular and macroscopic continuum descriptions of ion hydration. J. Phys. Chem. B 2000, 104, 7235-7238.

[58] Kalidas, C.; Hefter, G.; Marcus, Y. Gibbs Energies of Transfer of Cations from Water to Mixed Aqueous Organic Solvents. Chem. Rev. 2000, 100, 819-852.

[59] Joung, I. S.; Cheatham, T. E. Determination of Alkali and Halide Monovalent Ion Parameters for Use in Explicitly Solvated Biomolecular Simulations. J. Phys. Chem. B 2008, 112, 9020-9041.

[60] Gladich, I.; Shepson, P.; Szleifer, I.; Carignano, M. Halide and sodium ion parameters for modeling aqueous solutions in TIP5P-Ew water. Chem. Phys. Lett. 2010, 489, 113-117.

[61] Li, P.; Song, L. F.; Merz, K. M. Systematic parameterization of monovalent ions employing the nonbonded model. J. Chem. Theory Comput. 2015, DOI: 10.1021/ct500918t.

[62] Kelly, C. P.; Cramer, C. J.; Truhlar, D. G. Aqueous solvation free energies of ions and Ion-Water clusters based on an accurate value for the absolute aqueous solvation free energy of the proton. J. Phys. Chem. B 2006, 110, 16066-16081.

[63] Grycuk, T. Deficiency of the Coulomb-field approximation in the generalized Born model: An improved formula for Born radii evaluation. J. Chem. Phys. 2003, 119, 4817-4826.

[64] Chan, D. Y. C.; Mitchell, D. J.; Ninham, B. W. A model of solvent structure around ions. J. Chem. Phys. 1979, 70, 2946-2957.

[65] Mobley, D. L.; Dill, K. A.; Chodera, J. D. Treating entropy and conformational changes in implicit solvent simulations of small molecules. J. Phys. Chem. B 2008, 112, 938-946.

[66] Sanner, M. F.; Olson, A. J.; Spehner, J. C. Reduced surface: An efficient way to compute molecular surfaces. Biopolymers 1996, 38, 305-320.

[67] Nelder, J. A.; Mead, R. A simplex method for function minimization. Comput. J. 1965, 7, 308-313.

[68] Wang, J.; Wolf, R.; Caldwell, J.; Kollman, P.; Case, D. Development and testing of a general amber force field. J. Comput. Chem. 2004, 25, 1157-74.

[69] Wang, J.; Wang, W.; Kollman, P. A.; Case, D. A. Automatic atom type and bond type perception in molecular mechanical calculations. J. Mol. Graph. Model. 2006, 25, 247-260.

[70] Jakalian, A.; Jack, D. B.; Bayly, C. I. Fast, efficient generation of high-quality atomic charges. AM1-BCC model: II. Parameterization and validation. J Comput. Chem. 2002, 23, 1623-1641.

[71] Pronk, S.; Páll, S.; Schulz, R.; Larsson, P.; Bjelkmar, P.; Apostolov, R.; Shirts, M. R.; Smith, J. C.; Kasson, P. M.; van der Spoel, D.; Hess, E., Berk andn Lindahl GROMACS 4.5: a high-throughput and highly parallel open source molecular simulation toolkit. Bioinformatics 2013, 29, 845-854.

[72] Essmann, U.; Perera, L.; Berkowitz, M. L.; Darden, T.; Lee, H.; Pedersen, L. G. A smooth particle mesh Ewald method. J. Chem. Phys. 1995, 103, 8577-8593.

[73] Darden, T.; York, D.; Pedersen, L. Particle mesh Ewald: An N. $\log (\mathrm{N})$ method for Ewald sums in large systems. $J$. Chem. Phys. 1993, 98, 10089-10092. 